\title{
Ovomucoid-specific IgD increases in children who naturally outgrow egg allergy.
}

\author{
Naohiro Itoh ${ }^{1}$, Motoko Yasutomi ${ }^{1}$, Akiko Kawasaki ${ }^{1}$, Hiroki Murai ${ }^{1}$, Eishi Nomura ${ }^{2}$, Yuuki \\ Hagihara $^{3}$, Kazumasa Ogura ${ }^{4}$, and Yusei Ohshima ${ }^{1}$ \\ ${ }^{1}$ University of Fukui Faculty of Medical Sciences \\ ${ }^{2}$ Fukui Red Cross Hospital \\ ${ }^{3}$ Tsuruga City Hospital \\ ${ }^{4}$ Ohtaki Hospital
}

January 12, 2021

\section{Letter to Editor}

To the Editor

Egg-allergic children have higher ovomucoid (OVM)-specific $\operatorname{IgD}(\mathrm{sg} D)$ levels compared to the atopic controls. ${ }^{1}$ Within the egg-allergic group, children with higher levels of OVM-sIgD have a decreased risk of anaphylactic reactions. Ovalbumin (OVA)-sIgD increases in egg-allergic children desensitized by oral immunotherapy (OIT) but not in children unresponsive to OIT or with sustained unresponsiveness to OVA challenge. ${ }^{2}$ The natural development of tolerance and the acquisition of sustained unresponsiveness by OIT in egg-allergic children are associated with an increase in OVM-specific IgG4 (sIgG4) levels and a decrease in OVM-specific IgE (sIgE) levels. ${ }^{3}$ To elucidate the potential role of IgD in the outgrowing of egg allergy, we analyzed levels of egg white (EW)-, OVM-, and OVA-sIgD and sIgG4 in sera from 57 egg-allergic children (28 avoided all forms of egg in the diet (complete avoidance of egg: CAE), 18 were able to ingest at least 1/32 cooked whole egg but not one cooked whole egg (partial avoidance of egg: PAE), and 11 outgrew egg allergy (OGE)) and 23 healthy non-egg allergic children (non-egg allergy: NEA) (Table S1). The study was approved by The Research Ethics Committee of University of Fukui (\#20110052), and written informed consent was obtained from the parent or guardians.

EW-, and OVM-sIgE levels measured using ImmunoCAP (Thermo-Fisher Inc., MA) were higher in the CAE group, followed by the PAE, OGE, and NEA groups (Fig 1, Fig S1). The CAE group exhibited lower serum levels of EW- and OVA-sIgD compared to the NEA group and the PAE group, respectively, and had the lowest OVM-sIgD serum levels among all groups, suggesting that OVM-sIgD levels are associated with outgrowing egg allergy. We observed the lowest serum levels of EW-, OVA-, and OVM-sIgG4 in the CAE group, followed by the PAE and OGE groups. The ratio of OVM-sIgD to OVA-sIgD increased as children outgrew egg allergy, whereas the ratio of OVM-sIgG4 to OVA-sIgG4 did not change. Thus, the production of OVM-sIgD differs from OVM-sIgG4 as children naturally outgrow egg allergy.

High-affinity, but not low-affinity, IgE is known to cause anaphylaxis. ${ }^{4}$ High-affinity $\operatorname{IgE}$ is derived from memory $\operatorname{IgG1} 1^{+} \mathrm{B}$ cells, whereas low-affinity $\operatorname{IgE}$ is derived from naïve $\operatorname{IgM}^{+} \operatorname{IgD}^{+} \mathrm{B}$ cells. Considering class switching pathways, switching direction from IgM to IgD, and from IgG1 to IgE or IgG4, elevated OVM-sIgD levels might be associated with low-affinity OVM-sIgE levels as children outgrow egg allergy, resulting in hypo-responsiveness to OVM.

A recent study found that OVM-sIgE avidity was more effective at differentiating clinically reactive egg- 
allergic patients from those tolerant of heated egg compared to EW-sIgE. ${ }^{5}$ The ratio of OVM-sIgE to OVMsIgD or sIgG4 in the CAE group was significantly higher compared to the PAE, OGE, and NEA groups (Fig 2). Receiver operating analysis revealed that the ratio of OVM-sIgE to OVM-sIgD discriminated nontolerant from partially-tolerant egg-allergic patients with the largest area under the curve $(\mathrm{AUC}=0.965)$ compared with levels of OVM-sIgE or the ratio of OVM-sIgE to OVM-sIgG4. The optimal cutoff for the ratio of OVM-sIgE to OVM-sIgD had $86.5 \%$ sensitivity and $96.4 \%$ specificity to identify high-risk subjects (Table S2).

There are several limitations to this study. First, there was a small number of patients. Second, all children were only challenged with heated egg and were instructed to avoid egg of any form if they tested positive with less than $1 / 32$ cooked whole egg. Finally, there was a lack of trajectory of sIgD levels during natural tolerance development.

In conclusion, the ratio of OVM-sIgE to OVM-sIgD is a useful marker to identify high-risk egg-allergic patients capable of ingesting a low-dose of cooked whole egg who might be a good candidate for low-dose OIT.

\section{REFRENCES}

1. Suprun M, Getts R, Grishina G, et al. Ovomucoid epitope-specific repertoire of IgE, IgG4, IgG1, IgA1 , and IgD antibodies in egg-allergic children. Allergy. 2020;75:2633-2643. 2. Shan M, Carrillo J, Yeste A, et al. Secreted IgD Amplifies Humoral T Helper 2 Cell Responses by Binding Basophils via Galectin-9 and CD44.Immunity. 2018;49:709-724. 3. Caubet JC, Lin J, Ahrens B, et al. Natural tolerance development in cow's milk allergic children: IgE and IgG4 epitope binding. Allergy. 2017;72:1677-1685. 4. Gowthaman U, Chen JS, Eisenbarth SC. Regulation of IgE by T follicular helper cells. J Leukoc Biol. 2020;107:409-418. 5. Sato M, Yamamoto-Hanada K, Tada H, et al. Diagnostic performance of IgE avidity for hen's egg allergy in young infants. J Allergy Clin Immunol Pract. 2020;8:2417-2420.

Title: Ovomucoid-specific IgD increases in children who naturally outgrow egg allergy.

Short title: Ovomucoid-specific IgD in egg-allergic children

Authors: Naohiro Itoh $^{1}$, Motoko Yasutomi ${ }^{1}$, Akiko Kawasaki ${ }^{1}$, Hiroki Murai ${ }^{1}$, Eishi Nomura ${ }^{2}$, Yuuki Hagihara $^{3}$, Kazumasa Ogura ${ }^{4}$, and Yusei Ohshima ${ }^{1}$

\section{Affiliation}

1. Department of Pediatrics, Faculty of Medical Sciences, University of Fukui

2. Department of Pediatrics, Fukui Red Cross hospital

3. Department of Pediatrics, Municipal Tsuruga hospital

4. Department of Pediatrics, Ohtaki Hospital

\section{Corresponding Author}

Yusei Ohshima, M.D. Ph.D

Department of Pediatrics, Faculty of Medical Sciences, University of Fukui

23-3 Shimoaizuki, Matsuoka, Yoshida-gun, Fukui 910-1193, JAPAN

Tel: +81-776-61-3111, ext.2316 Fax: +81-776-61-8129 e-mail:yohshima@u-fukui.ac.jp

\section{Key words:}

egg white, food allergy, $\operatorname{IgD}, \operatorname{IgG} 4$, ovomucoid

\section{Conflict of interest:}

The authors declare that they have no conflicts of interest.

\section{Acknowledgements}


We thank Ms. Michiyo Toyooka and Ms. Yuki Mori for their assistance in carrying out Ig measurement.

\section{Author contribution:}

NI, MY, AK, HM, EN, YH, and KO collected the samples. NI wrote the manuscript and YO organized the study. All authors read and approved the final manuscript. This work was supported in part by a Grant-in-Aid for Scientific Research from the Japan Society for the Promotion of Science (to YO).

\section{Figure legends}

Figure 1 Egg allergen-specific IgE, IgD, and IgG4 levels in egg-allergic and non-egg allergic patients (NEA). Ovomucoid (OVM)-specific IgE (sIgE)(A), specific $\operatorname{IgD}(\operatorname{sIgD})(\mathrm{B})$, and specific $\operatorname{IgG} 4(\mathrm{sIgG} 4)(\mathrm{C})$, and relationship between ovalbumin (OVA) and OVM-sIgD (D) or sIgG4 (E) in the complete avoidance of egg (CAE), partial avoidance of egg (PAE), outgrown egg allergy (OGE), and NEA groups. ${ }^{*} \mathrm{p}<0.05,{ }^{* *} \mathrm{p}<0.01$, $* * * \mathrm{p}<0.001$

Figure 2 Relationship between ovomucoid-specific IgD, IgG4, and IgE in egg-allergic and non-egg allergic patients (NEA), and Receiver-operating characteristic predicting tolerance to cooked egg white ingestion. The logarithms of the ratio of OVM-sIgE to OVM-sIgD (A), OVM-sIgE to OVM-sIgG4 (B), and OVMsIgD to OVM-sIgG4 in the complete avoidance of egg (CAE), partial avoidance of egg (PAE), outgrown egg allergy (OGE), and NEA groups. Receiver-operating characteristic curves (C) of OVM-sIgE (long dashed double-dotted line), sIgE/sIgG4 (dotted line), sIgD (broken line), and sIgE/sIgD (bold line).

${ }^{*} \mathrm{p}<0.001$

\section{Hosted file}

Figure.pdf available at https ://authorea.com/users/388854/articles/503586-ovomucoid-specificigd-increases-in-children-who-naturally-outgrow-egg-allergy 